Research Article

\title{
Comparative Analysis of Clinical and Medication Information between Chronic Hepatitis B Patients with Damp Heat Syndrome and Spleen Deficiency Syndrome
}

\author{
Qiao-Hong Liu $\mathbb{D},{ }^{1}$ Bin-Bin Zhang $\mathbb{D}^{1},{ }^{1}$ Lin Xu $\mathbb{D}^{1},{ }^{1}$ Xiao-Ping Shen $\mathbb{D}^{1},{ }^{1}$ Ya-Mei Hai $\mathbb{D}$, \\ Yi-Yang $\mathrm{Hu}\left(\mathbb{1},,^{2,3}\right.$ and Yu Zhao $\mathbb{C}^{1,3}$ \\ ${ }^{1}$ Key Laboratory of Liver and Kidney Diseases, Institute of Liver Diseases, \\ Shuguang Hospital Affiliated to Shanghai University of Traditional Chinese Medicine, Shanghai 201203, China \\ ${ }^{2}$ Institute of Clinical Pharmacology, Shuguang Hospital Affiliated to Shanghai University of Traditional Chinese Medicine, \\ Shanghai 201203, China \\ ${ }^{3}$ Shanghai Key Laboratory of Traditional Chinese Clinical Medicine, Shanghai 201203, China \\ Correspondence should be addressed to Yi-Yang Hu; yyhuliver@163.com and Yu Zhao; cathy150@139.com
}

Received 15 September 2020; Revised 9 December 2020; Accepted 18 December 2020; Published 28 December 2020

Academic Editor: Vincenzo De Feo

Copyright (c) 2020 Qiao-Hong Liu et al. This is an open access article distributed under the Creative Commons Attribution License, which permits unrestricted use, distribution, and reproduction in any medium, provided the original work is properly cited.

\begin{abstract}
Traditional Chinese medicine (TCM) has a long history in the treatment of chronic hepatitis B (CHB) based on the syndrome identification. Previous studies reported CHB patients with damp-heat (DH) syndrome accompanied with a severe liver function damage, but lacked the medication analysis. In this study, we analyzed $999 \mathrm{CHB}$ patients with unidentified individual-level data from database to explore clinical features of two common syndromes of $\mathrm{CHB}$ patients based on the real world. Compared with the spleen deficiency (SD) syndrome, the $\mathrm{CHB}$ patients with $\mathrm{DH}$ syndrome had a significantly higher level of alanine aminotransferase $($ ALT) and aspartate aminotransferase (AST) $(P<0.05)$ but took more immunomodulators and hepatoprotective drugs $(P<0.1)$. Similarly, in the follow-up of 207 patients after 3 months, the improvement trend of ALT and AST of patients with sustained SD syndrome was significantly better than those whose TCM syndrome changed from SD to DH $(P<0.05)$. The logistic model indicated DH syndrome was a significant negative factor for reducing ALT level in CHB patients $(\mathrm{OR}=4.854, P=0.032)$. This study suggests that $\mathrm{CHB}$ patients with $\mathrm{DH}$ syndrome have potentially more serious and sustained liver damage than the SD syndrome, which provides a reference for the personalized management of CHB patients from the perspective of TCM syndromes.
\end{abstract}

\section{Introduction}

Hepatitis B is caused by hepatitis B virus (HBV) infection. At present, the prevalence of hepatitis $B$ surface antigen (HBsAg)-positive is about $4.9 \%$ worldwide [1]. China has a high incidence of hepatitis $B$. It is estimated that the prevalence of $\mathrm{HBV}$ infection in the general population of China is $5 \%-7.99 \%$ [2]. About $50 \%$ patients with HCC were complicated with HBV infection [3]; the Global Hepatitis Report 2017 shows that approximately 887 thousand people die of chronic hepatitis $\mathrm{B}$ - (CHB-) related complications worldwide in 2015, mainly due to liver cirrhosis and hepatocellular carcinoma. To prevent the prognosis of $\mathrm{CHB}$, early diagnosis and effective treatment are very important.

The current treatment of $\mathrm{CHB}$ is mainly antiviral drugs, which can effectively block the replication of HBV-DNA and reduce the incidence of cirrhosis and HCC [4]. Traditional Chinese medicine (TCM) is widely used in China; it has the advantages of early treatment and combined intervention in the treatment of hepatitis $B$, which is reported to be beneficial for alleviating liver damage, reducing jaundice, regulating immunity [5], and inhibiting the development of liver fibrosis and HCC. TCM also was reported to lower the risk of death among $\mathrm{CHB}$ patients with contaminant liver cirrhosis and 
HCC [6-8]. Different from the strict indication screening of antiviral therapy, TCM can alleviate physical uncomfortable symptoms and improve the quality of life of $\mathrm{CHB}$ patients who are not suitable for antiviral therapy but have somatic discomfort symptoms such as pain in the liver area.

The characteristic of TCM is to provide personalized treatment based on the theory of Chinese medicine, in which the differentiation of TCM syndromes is the premise of personalized treatment. Since TCM and western medicine have common treatment subjects, whether there are clinical differences based on modern laboratory indicators in different syndromes has always been the focus of syndrome research. The damp-heat $(\mathrm{DH})$ syndrome and spleen deficiency (SD) syndrome are the main subtypes of $\mathrm{CHB}$ according to the TCM syndrome differentiation $[9,10]$. DH syndrome is reported to be accompanied by a higher liver injury in most of the literatures, but the liver function is easily interfered by medication in the real world. For example, some patients will quickly recover from liver damage after taking liver protection drugs. Existing studies compared the expression of liver function in patients with different syndromes but payed little attention to the usage of drugs [11-14], which cannot rule out the potential interference by the difference in medications. Besides, the TCM syndromes are not immutable; the dynamic longitudinal observation is necessary. By dynamically observing the changes in syndromes and combining with the medication, the clinical characteristics of different syndromes of $\mathrm{CHB}$ can be further clarified. Therefore, this study will analyze the clinical differences of two common TCM syndromes of $\mathrm{CHB}$; we first explore the clinical indexes and medication differences between syndromes in a cross-sectional study and then confirm the clinical differences by longitudinal dynamic study analysis.

\section{Methods}

2.1. Study Design. All procedures followed were in accordance with the ethical standards of the responsible committee on human experimentation (institutional and national) and with the Helsinki Declaration of 1975, as revised in 2000 (5), and were approved by the IRB of Shuguang Hospital affiliated to Shanghai University of Traditional Chinese Medicine (Permit Number: 2012-20622-01). Informed consent was obtained from all patients for being included in the study. This is a cross section study based on 2079 unidentified individual-level data from the database collected from 2008 to 2015, which consisted of two parts. The first data set contained $999 \mathrm{CHB}$ patients with TCM syndrome differentiation records in the database; we described the distribution of syndrome types and compared the general information, clinical indicators, and drug usage between $\mathrm{CHB}$ patients with DH syndrome and SD syndrome. Among the patients in the first data set, $207 \mathrm{CHB}$ patients were followed up after 3 months to form data set 2 . We recorded the changes in the syndrome of these patients, compared the clinical indicators and medication information, and analyzed the related risk factors that affect the improvement of ALT (Figure 1).
2.2. Inclusion and Exclusion Criteria of Participants. Patients who met the diagnostic criteria of "chronic hepatitis B" [15] according to the "China Guidelines for Prevention and Treatment of Chronic Hepatitis B (2005)” [16], aged from 18 to 65, and signed informed consent were included. Patients who had viral hepatitis, chronic severe hepatitis, severe primary diseases, or mental diseases or were pregnant women or lactating women were excluded. The standard of the TCM syndrome differentiation was according to the "TCM Syndrome Differentiation Standard of Viral Hepatitis" [17] and "The Clinical Research Guide of New Drugs of Traditional Chinese Medicine" [18]; detailed syndrome differentiation standards are attached in Additional file 2.

2.3. Statistics. We used SPSS (version 26.0) for all statistical analyses. Shapiro-Wilk test was used to assess data normality. We evaluated the numerical variables that did not violate normality test and homogeneity test of variance by $T$ test, while the remaining numerical variables were compared using the Mann-Whitney $U$-test, and categorical data adopted the $\chi^{2}$ test. The normal distribution variables were expressed as mean \pm standard deviation $(x \pm s)$; the nonnormal distribution variables were expressed using the median (median (1/4 quantile, 3/4 quantile)). The correlation analysis was estimated by the logistic regression. Simple logistic regression was conducted to estimate crude odds ratios (OR) and their 95\% confidence intervals (95\% CI) for all factors related to ALT differences. Multiple logistic regression was used to adjust potential confounding by age, gender, and other drug use. The differences were considered significant at $P<0.05$.

\section{Results}

3.1. Comparison of Clinical Indexes and Medication of $\mathrm{CHB}$ Patients with DH Syndrome and SD Syndrome at Baseline. Among the $999 \mathrm{CHB}$ patients, the proportion of $\mathrm{DH}$ syndrome was the highest (\%), followed by the SD syndrome (\%) (Figure 2(a)). We chose CHB patients with DH syndrome $(n=429)$ or SD syndrome $(n=383)$ to analyze the clinical indexes differences. The ALT, AST, TBA, and PT values of the $\mathrm{DH}$ syndrome were significantly higher than those of the SD syndrome $(P=0.048, P=0.024, P=0.006$, $P=0.002$, respectively). The age and AFP values of patients with $\mathrm{DH}$ syndrome were slightly higher than those of patients with SD syndrome, but there was no statistical difference (Table 1). To assess the effect of drugs on differences in liver function, we compared the clinical medications of the two groups of patients. There was no difference in the composition of antiviral drugs, Chinese herbal medicine, and antifibrosis proprietary Chinese medicine between $\mathrm{DH}$ syndrome and SD syndrome. However, the proportion of immunomodulators in the $\mathrm{DH}$ syndrome group was significantly higher than that in the SD syndrome group $(P<0.05)$. And the proportion of hepatoprotective drugs and drugs that promote bile excretion of the $\mathrm{DH}$ syndrome was slightly higher than that in the SD syndrome $(P<0.1$, Table 1). 


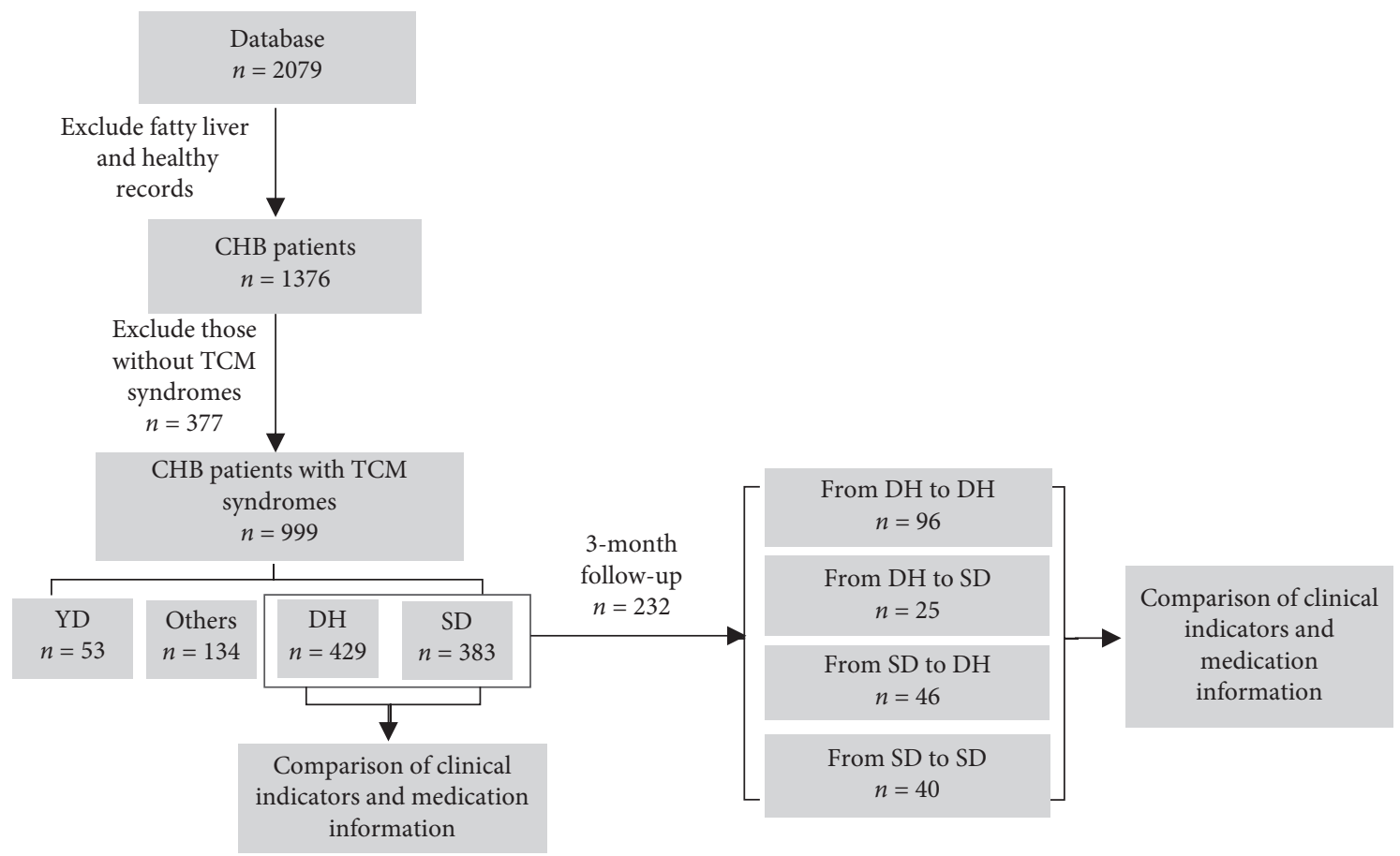

Figure 1: Study design.

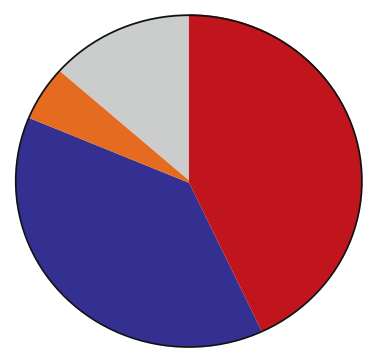

Total $=999$

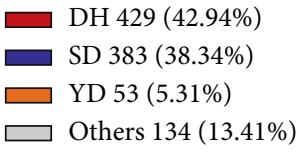

(a)

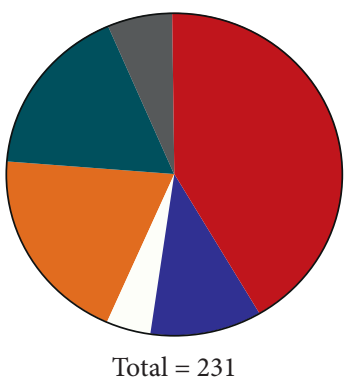

DH-DH 96 (41.56\%)

DH-SD 25 (10.82\%)

DH-others $9(3.90 \%)$

SD-DH 46 (19.91\%)

SD-SD 40 (17.32\%)

SD-others 15 (6.49\%)

(b)

FIgUre 2: The syndrome distribution in $999 \mathrm{CHB}$ patients and the syndrome conversion after 3-month follow-up. (a) Distribution of TCM syndrome in $999 \mathrm{CHB}$ cases. (b) Conversion of syndrome types in $231 \mathrm{CHB}$ patients after 3-month follow-up.

3.2. Comparison of Clinical Indexes and Drug Use among the Four Main TCM Syndrome Conversion Groups in $\mathrm{CHB} \mathrm{Pa}$ tients after 3-Month Follow-Up. Among the $232 \mathrm{CHB}$ patients who were followed up after 3 months, the composition ratio of syndrome changes after 3 months is shown in Figure 1(b). Among the 130 patients diagnosed with $\mathrm{DH}$ syndrome for the first time, $96(73.84 \%)$ patients maintained the DH syndrome unchanged (group A), and 25 (19.23\%) patients changed from DH syndrome to SD syndrome after 3 months (group B). Among the 101 patients diagnosed with SD syndrome at the first time point, 46 (45.54\%) patients changed to $\mathrm{DH}$ syndrome after 3 months of follow-up (group C), and 40 (39.60\%) patients maintained the SD syndrome unchanged (group D).

We compared the dynamic differences in clinical indicators between patients with syndrome changes and those with unchanged syndromes. As seen in Table 2, there was no significant difference in gender, age, and medication between patients in groups $C$ and $D$. Compared with group $C$, the improvement of TBIL, AST, and ALB in group D was more significant $(P<0.05)$, and the improvement trend of ALT, APRI, and FIB-4 in group D was slightly better than that in group $\mathrm{C}(P<0.1)$. The results indicate that $\mathrm{CHB}$ patients with SD syndrome who converted into $\mathrm{DH}$ 
TABLE 1: Baseline analysis of 812 CHB patients with DH syndrome and the SD syndrome.

\begin{tabular}{|c|c|c|c|c|}
\hline & $\mathrm{DH}(n=429)$ & $\mathrm{SD}(n=383)$ & Statistics & $P$ \\
\hline Gender (male, \%) & $326(75.99 \%)$ & $213(55.61 \%)$ & $0.889^{\mathrm{a}}$ & 0.346 \\
\hline Age (years) & $44.44(37.1,54.61)$ & $44.06(36.22,51.38)$ & -1.887 & 0.059 \\
\hline \multicolumn{5}{|l|}{ Treatment } \\
\hline Antiviral therapy & $259(60.37 \%)$ & $231(60.31 \%)$ & $0.000^{\mathrm{a}}$ & 0.986 \\
\hline Recent anti-virus* & $60(23.34 \%)$ & $51(22.76 \%)$ & $0.023^{\mathrm{a}}$ & 0.881 \\
\hline Antivirus duration (days) & $325(96,758.5)$ & $370.5(101,1074.5)$ & -1.378 & 0.168 \\
\hline Hepatoprotective drugs & $258(60.13 \%)$ & $253(66.05 \%)$ & 3.037 & 0.081 \\
\hline Immunomodulator & $15(3.49 \%)$ & $4(1.04 \%)$ & $5.324^{\mathrm{a}}$ & 0.021 \\
\hline Drugs that promote bile excretion & $46(10.72 \%)$ & $28(7.31 \%)$ & $2.844^{\mathrm{a}}$ & 0.092 \\
\hline Chinese herbal medicine & $108(25.65 \%)$ & $99(25.84 \%)$ & $0.048^{\mathrm{a}}$ & 0.826 \\
\hline Anti-fibrosis proprietary Chinese medicine & $18(4.19 \%)$ & $21(5.48 \%)$ & $0.733^{\mathrm{a}}$ & 0.392 \\
\hline Untreated & $81(18.88 \%)$ & $70(18.27 \%)$ & $0.049^{\mathrm{a}}$ & 0.825 \\
\hline \multicolumn{5}{|l|}{ Liver function value } \\
\hline TBIL $(\mu \mathrm{mol} / \mathrm{L})$ & $16.6(12.5,22.47)$ & $15.7(12.47,21.08)$ & -1.119 & 0.263 \\
\hline DBIL $(\mu \mathrm{mol} / \mathrm{L})$ & $4.4(3.3,6.7)$ & $4.4(3.03,6.3)$ & -0.994 & 0.32 \\
\hline $\operatorname{ALT}(\mathrm{U} / \mathrm{L})$ & $44(26,92.5)$ & $40.8(23.3,79.75)$ & -1.977 & 0.048 \\
\hline $\operatorname{AST}(\mathrm{U} / \mathrm{L})$ & $36(26.02,60.75)$ & $33(24,56)$ & -2.25 & 0.024 \\
\hline GGT (U/L) & $29.85(18,65.5)$ & $28(17.77,55.25)$ & -1.352 & 0.176 \\
\hline $\operatorname{ALP}(\mathrm{U} / \mathrm{L})$ & $79(64,100)$ & $75.15(63,96)$ & -1.617 & 0.106 \\
\hline $\operatorname{ALB}(g / L)$ & $45.5(43,47.8)$ & $45.4(42.62,48.1)$ & -0.315 & 0.753 \\
\hline Pre-Alb (g/L) & $241(178,290)$ & $237.35(180,288.5)$ & -0.043 & 0.966 \\
\hline $\mathrm{TBA}(\mu \mathrm{mol} / \mathrm{L})$ & $7.6(3.9,14)$ & $5.9(2.9,12.8)$ & -2.749 & 0.006 \\
\hline \multicolumn{5}{|l|}{ Hepatitis B virus } \\
\hline HBeAg positive (\%) & $227(53.53 \%)$ & $186(49.33 \%)$ & $1.410^{\mathrm{a}}$ & 0.235 \\
\hline HBV-DNA (IU/mL) & $13020(0,2270000)$ & $7181(0,2115000)$ & -1.245 & 0.213 \\
\hline \multicolumn{5}{|l|}{ Liver fibrosis value } \\
\hline APRI & $0.48(0.31,0.91)$ & $0.46(0.27,0.80)$ & -1.429 & 0.153 \\
\hline FIB-4 & $1.63(1.07,2.77)$ & $1.52(1.01,2.33)$ & -1.603 & 0.109 \\
\hline \multicolumn{5}{|l|}{ Other biomarkers } \\
\hline $\mathrm{PT}(\mathrm{S})$ & $13.2(12.2,14.4)$ & $12.7(12,13.9)$ & -3.054 & 0.002 \\
\hline $\operatorname{AFP}(n g / m L)$ & $3.67(2.6,6.5)$ & $3.45(2.36,5.84)$ & -1.845 & 0.065 \\
\hline $\mathrm{CD} 4 / \mathrm{CD} 8$ & $1.33(1.02,1.76)$ & $1.26(0.89,1.73)$ & -1.641 & 0.101 \\
\hline \multicolumn{5}{|l|}{ Lipids, Blood glucose } \\
\hline $\mathrm{FBG}(\mathrm{mmol} / \mathrm{L})$ & $5.18(4.85,5.47)$ & $5.14(4.85,5.5)$ & -0.601 & 0.548 \\
\hline TC $(\mathrm{mmol} / \mathrm{L})$ & $4.59(3.83,5.2)$ & $4.58(3.96,5.22)$ & -0.891 & 0.373 \\
\hline TG $(\mathrm{mmol} / \mathrm{L})$ & $1.1(0.82,1.49)$ & $1.07(0.79,1.52)$ & -0.281 & 0.779 \\
\hline HDL-C (mmol/L) & $1.18(1,1.415)$ & $1.2(1.01,1.43)$ & -0.666 & 0.506 \\
\hline $\mathrm{LDL}-\mathrm{C}(\mathrm{mmol} / \mathrm{L})$ & $2.54(2.02,2.94)$ & $2.46(2.11,3.05)$ & -0.611 & 0.541 \\
\hline \multicolumn{5}{|l|}{ Blood routine test } \\
\hline $\mathrm{WBC}\left(10^{9} / \mathrm{L}\right)$ & $5.01(4.26,6.01)$ & $5(4.13,6.04)$ & -0.259 & 0.796 \\
\hline $\operatorname{LY}\left(10^{9} / \mathrm{L}\right)$ & $1.64(1.28,2)$ & $1.68(1.32,2.04)$ & -0.656 & 0.512 \\
\hline MONO $\left(10^{9} / \mathrm{L}\right)$ & $0.31(0.24,0.4)$ & $0.32(0.26,0.41)$ & -0.769 & 0.442 \\
\hline NEUT $\left(10^{9} / \mathrm{L}\right)$ & $2.89(2.25,3.6)$ & $2.8(2.16,3.65)$ & -0.749 & 0.454 \\
\hline PLT $\left(10^{9} / \mathrm{L}\right)$ & $159(124,202.5)$ & $159(122,198)$ & -0.151 & 0.88 \\
\hline \multicolumn{5}{|l|}{ Renal function value } \\
\hline BUN $(\mathrm{mmol} / \mathrm{L})$ & $4.48(3.61,5.2)$ & $4.37(3.6,5.11)$ & -0.718 & 0.473 \\
\hline $\mathrm{Cr}(\mu \mathrm{mol} / \mathrm{L})$ & $70(59.78,80)$ & $68(60,78)$ & -1.253 & 0.21 \\
\hline $\mathrm{UA}(\mu \mathrm{mol} / \mathrm{L})$ & $300(256.5,354)$ & $303.5(244,352)$ & -0.37 & 0.712 \\
\hline
\end{tabular}

* The time interval between the start date of antiviral treatment and the enrollment date $\leq 90$ days. APRI $=(\mathrm{AST}(\mathrm{U} / \mathrm{L}) / \mathrm{upper}$ limit of normal value $(\mathrm{U} / \mathrm{L})) / \mathrm{PLT}$ $\left(\times 10^{9} / \mathrm{L}\right) \times 100$; FIB- $4=$ age $($ year $) \times$ AST $(\mathrm{U} / \mathrm{L}) /\left(\mathrm{PLT}\left(\times 10^{9} / \mathrm{L}\right) \times \mathrm{ALT}(\mathrm{U} / \mathrm{L})^{1 / 2}\right)$.

syndrome have a worse clinical recovery of liver function than those who maintained SD syndrome, which is consistent with the trend of liver damage in the first crosssectional observation. Although no significant difference was found in the clinical indicator comparation between group $\mathrm{A}$ and group $\mathrm{B}$, the $\mathrm{CHB}$ patients who have maintained $\mathrm{DH}$ syndrome had a worse liver function trend than those of SD syndrome patients. In terms of medication, the untreated patient in group A was significantly higher than that in group $\mathrm{B}$, and the proportion of taking Chinese herbal medicine in group A was significantly lower than that in group B. An additional file shows this in more detail (see Additional file 1).

3.3. Analysis of the Factors Related to the Improvement of ALT Level in CHB Patients. We used the logistic regression model to analyze whether TCM syndrome is a variable closely 
TABLE 2: Comparison of clinical index difference and medication between group C and group D.

\begin{tabular}{|c|c|c|c|c|}
\hline & Group C (SD to DH) $n=46$ & Group D (sustained SD) $n=40$ & Statistics & $P$ \\
\hline $\begin{array}{l}\text { Gender (male, \%) } \\
\text { Age (years) }\end{array}$ & $\begin{array}{c}32(69.56 \%) \\
41.64(36.54,51.49) \\
\end{array}$ & $\begin{array}{c}32(80 \%) \\
48.48(37.64,51.98) \\
\end{array}$ & $\begin{array}{c}1.224^{\mathrm{a}} \\
-1.056 \\
\end{array}$ & $\begin{array}{l}0.269 \\
0.291 \\
\end{array}$ \\
\hline $\begin{array}{l}\text { Treatment } \\
\text { Antiviral therapy } \\
\text { Recent antivirus } \\
\text { Antivirus duration (days) } \\
\text { Hepatoprotective drugs } \\
\text { Immunomodulator } \\
\text { Drugs that promote bile excretion } \\
\text { Chinese herbal medicine } \\
\text { Antifibrosis proprietary Chinese medicine } \\
\text { Untreated }\end{array}$ & $\begin{array}{c}26(56.52 \%) \\
10(21.73 \%) \\
116(53.5,515) \\
18(39.13 \%) \\
0(0 \%) \\
4(8.69 \%) \\
17(36.95 \%) \\
1(2.2 \%) \\
4(8.69 \%)\end{array}$ & $\begin{array}{c}28(70 \%) \\
9(22.5 \%) \\
173(53.5,416) \\
19(47.5 \%) \\
0(0 \%) \\
3(7.5 \%) \\
15(37.5 \%) \\
3(7.5 \%) \\
0(0 \%)\end{array}$ & $\begin{array}{c}1.664^{\mathrm{a}} \\
0.007^{\mathrm{a}} \\
-0.090 \\
0.611^{\mathrm{a}} \\
- \\
0.041^{\mathrm{a}} \\
0.003^{\mathrm{a}} \\
1.369^{\mathrm{a}} \\
3.648^{\mathrm{a}}\end{array}$ & $\begin{array}{l}0.197 \\
0.932 \\
0.928 \\
0.434 \\
- \\
0.579 \\
0.959 \\
0.257 \\
0.077\end{array}$ \\
\hline $\begin{array}{l}\text { Liver function value } \\
\text { TBIL }(\mu \mathrm{mol} / \mathrm{L}) \\
\text { DBIL }(\mu \mathrm{mol} / \mathrm{L}) \\
\text { ALT }(\mathrm{U} / \mathrm{L}) \\
\text { AST }(\mathrm{U} / \mathrm{L}) \\
\text { GGT }(\mathrm{U} / \mathrm{L}) \\
\text { ALP }(\mathrm{U} / \mathrm{L}) \\
\text { ALB }(\mathrm{g} / \mathrm{L}) \\
\text { Pre-Alb }(\mathrm{g} / \mathrm{L}) \\
\text { TBA }(\mu \mathrm{mol} / \mathrm{L})\end{array}$ & $\begin{array}{c}0.04(-1.87,3.14) \\
0(-0.7,1) \\
-11(-34,5.75) \\
-2(-15.25,9.75) \\
-1(-23,3) \\
0.5(-11.25,8.25) \\
1(-2.32,2.82) \\
0(-112,64.5) \\
-2.3(-5.05,0.02) \\
\end{array}$ & $\begin{array}{c}-1.6(-6.65,2.22) \\
0(-1.72,1.12) \\
-19(-96,-6.25) \\
-12.5(-69,1) \\
-8.5(-51,3) \\
-3(-13,8.75) \\
2.19(0.05,5.15) \\
19(-116.5,70) \\
-2.75(-15.07,-0.27)\end{array}$ & $\begin{array}{l}-1.979 \\
-0.792 \\
-1.875 \\
-2.130 \\
-1.178 \\
-0.723 \\
-2.156 \\
-0.004 \\
-1.130 \\
\end{array}$ & $\begin{array}{l}\mathbf{0 . 0 4 8} \\
0.428 \\
0.061 \\
\mathbf{0 . 0 3 3} \\
0.239 \\
0.469 \\
\mathbf{0 . 0 3 1} \\
0.996 \\
0.258\end{array}$ \\
\hline $\begin{array}{l}\text { Hepatitis B virus } \\
\text { HBeAg (S/CO) } \\
\text { HBV-DNA (IU/mL) }\end{array}$ & $\begin{array}{r}-0.075(-155.5925,0.775) \\
-35280(-2775275,643.75) \\
\end{array}$ & $\begin{array}{c}-3.135(-90.502,0.385) \\
-25080.5(-5387429.25,0) \\
\end{array}$ & $\begin{array}{c}0.444^{\mathrm{a}} \\
-0.320 \\
\end{array}$ & $\begin{array}{l}0.505 \\
0.749 \\
\end{array}$ \\
\hline $\begin{array}{l}\text { Liver fibrosis value } \\
\text { FIB-4 } \\
\text { APRI }\end{array}$ & $\begin{array}{c}0.04(-0.21,0.36) \\
-0.02(-0.1826,0.069) \\
\end{array}$ & $\begin{array}{l}-0.22(-0.86,0.42) \\
-0.18(-0.83,0.02) \\
\end{array}$ & $\begin{array}{l}-1.697 \\
-1.939 \\
\end{array}$ & $\begin{array}{c}0.09 \\
0.052 \\
\end{array}$ \\
\hline $\begin{array}{l}\text { Other biomarkers } \\
\text { PT }(\mathrm{S}) \\
\text { AFP }(\mathrm{ng} / \mathrm{mL}) \\
\text { CD4/CD8 }\end{array}$ & $\begin{array}{c}-0.24(-0.82,0.52) \\
-1.13(-3.50,0.05) \\
0.06(-0.18,0.37) \\
\end{array}$ & $\begin{array}{c}0(-0.75,0.67) \\
-0.97(-3.18,-0.05) \\
0.07(-0.13,0.31) \\
\end{array}$ & $\begin{array}{l}-0.671 \\
-0.089 \\
-0.320\end{array}$ & $\begin{array}{l}0.502 \\
0.929 \\
0.749 \\
\end{array}$ \\
\hline $\begin{array}{l}\text { Lipids, blood glucose } \\
\text { FBG (mmol/L) } \\
\text { TC (mmol/L) } \\
\text { TG }(\mathrm{mmol} / \mathrm{L}) \\
\text { HDL-C }(\mathrm{mmol} / \mathrm{L}) \\
\text { LDL-C }(\mathrm{mmol} / \mathrm{L})\end{array}$ & $\begin{array}{c}-0.09(-0.51,0.06) \\
-0.27(-0.73,0.13) \\
-0.03(-0.26,0.13) \\
-0.16 \pm 0.33 \\
-0.10 \pm 0.69\end{array}$ & $\begin{array}{c}0.24(-0.03,0.50) \\
-0.32(-1.30,0.2) \\
-0.08(-0.57,0.19) \\
-0.09 \pm 0.51 \\
-0.31 \pm 1.04\end{array}$ & $\begin{array}{c}-3.355 \\
-0.377 \\
-0.610 \\
-0.818 \\
1.103 \\
\end{array}$ & $\begin{array}{l}\mathbf{0 . 0 0 1} \\
0.706 \\
0.542 \\
0.416 \\
0.273 \\
\end{array}$ \\
\hline $\begin{array}{l}\text { Blood routine test } \\
\text { WBC }\left(10^{9} / \mathrm{L}\right) \\
\text { LY }\left(10^{9} / \mathrm{L}\right) \\
\text { MONO }\left(10^{9} / \mathrm{L}\right) \\
\text { NEUT }\left(10^{9} / \mathrm{L}\right) \\
\text { PLT }\left(10^{9} / \mathrm{L}\right)\end{array}$ & $\begin{array}{c}0.14 \pm 1.19 \\
0.04 \pm 0.40 \\
0.01(-0.10,0.10) \\
0.11(-0.4,0.71) \\
-4.59 \pm 27.25\end{array}$ & $\begin{array}{c}0.39 \pm 1.23 \\
0.07 \pm 0.53 \\
-0.02(-0.16,0.08) \\
0.55(-0.19,1.23) \\
5.63 \pm 31.57\end{array}$ & $\begin{array}{l}-0.964 \\
-0.306 \\
-0.737 \\
-1.367 \\
-1.610\end{array}$ & $\begin{array}{c}0.338 \\
0.76 \\
0.461 \\
0.172 \\
0.111\end{array}$ \\
\hline
\end{tabular}

related to a different recovery of serum ALT. Since the serum ALT level of most patients in the follow-up after 3 months was lower than the first time point, we used whether the ALT decreased more than 30\% from the baseline level during the follow-up after 3 months as the dependent variable. To exclude the interference of the syndrome conversion, $\mathrm{CHB}$ patients in group A or group D whose serum ALT level was abnormal (more than 50U/L) at baseline were included in the model (including 49 patients in group A and 24 patients in group D). Simple logistic regression analysis was used to include syndrome grouping and medication information as independent variables. DH syndrome is a significant negative factor (OR [95\% CI]: 4.854 [1.149-20.501], $P=0.032$ ).
Then, we used the multiple logistic regression model, which included age, gender, and HBV-DNA value as covariates; the adjusted OR value of DH syndrome was $4.936(P=0.032)$. These results indicate that DH syndrome is a negative factor for reducing the serum ALT level in CHB patients (Table 3).

\section{Discussion}

In this study, we analyzed clinical data of $999 \mathrm{CHB}$ patients with TCM syndrome records. Among these patients, DH syndrome and SD syndrome were the two most common syndromes, accounting for $81.72 \%$ of the total collected $\mathrm{CHB}$ patients, which was consistent with previous research 
TABLE 3: Analysis of factors related to the decrease of ALT.

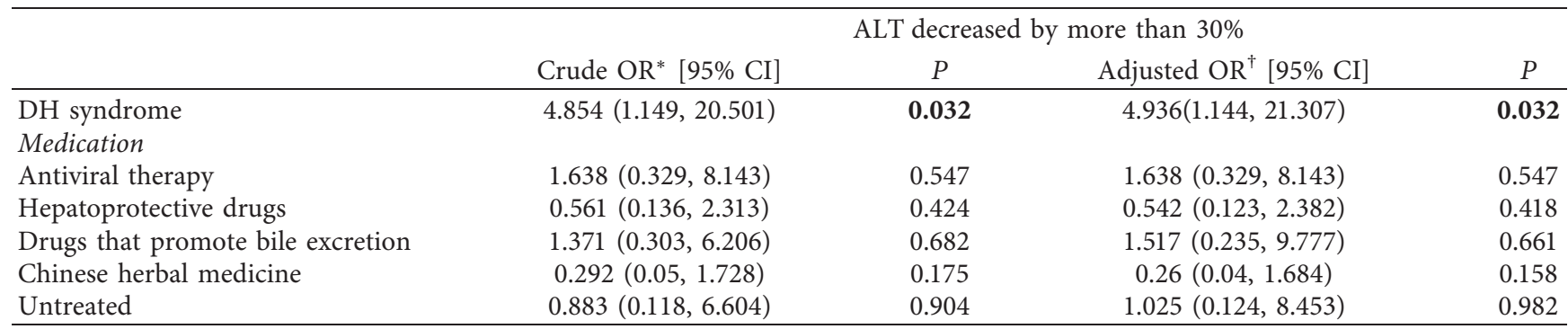

Analysis of factors related to the decrease of ALT in patients with ALT $>50 \mathrm{U} / \mathrm{L}$ at baseline of group A\&D. ${ }^{*}$ Crude OR estimated by the simple logistic regression model. ${ }^{\dagger}$ Adjusted OR estimated by multiple logistic regression models including age, gender, and HBV-DNA value as covariates.

reports $[10,19]$. In our study, the ALT and AST levels of patients with $\mathrm{DH}$ syndrome were significantly higher than those of the SD group $(P=0.048, P=0.024$, respectively), indicating that the liver damage of $\mathrm{CHB}$ patients with $\mathrm{DH}$ syndrome was higher than that of SD syndrome, which is consistent with the results of previous reports that $\mathrm{CHB}$ patients with DH syndrome have higher levels of ALT or AST [11-14]. Besides, some studies have pointed out that the ALT and AST levels of autoimmune hepatitis patients with $\mathrm{DH}$ syndrome were significantly higher than those of SD syndrome [20]; patients of NAFLD with DH syndrome also had a significantly higher ALT level than that of other syndrome types [21]. Therefore, we speculate that patients with $\mathrm{DH}$ syndrome have a higher level of liver damage which is a common manifestation of chronic liver diseases.

Because HBV is a noncytopathic virus, liver injury is mediated by the immune-mediated cytotoxicity in chronic infection. Therapeutic control of HBV-DNA and ALT in $\mathrm{CHB}$ is associated with decreased NK cell killing of HBVpositive hepatocytes and $\mathrm{HBV}$-specific T cells, and increased antiviral T-cell response [22]. In dynamic follow-up, we hope to observe whether patients with different syndromes of $\mathrm{CHB}$ also have differences in drug response and disease outcomes. Due to drug intervention, the level of ALT and AST was decreased in varying degrees in most of the followup dynamic observation patients, but the improvement trend of TBIL, ALT, and AST of CHB patients with sustained SD syndrome was significantly better than those whose TCM syndrome changed from SD to DH $(P=0.048, p=0.061$, $p=0.033$, respectively). Similarly, patients with $\mathrm{CHB} \mathrm{DH}$ syndrome converted to SD syndrome had a better recovery trend of liver damage than patients with persistent $\mathrm{DH}$ syndrome but without statistical difference, which might be related to the imbalanced number of cases between the two groups. As seen in Supplementary Table 1, compared with group A, the difference of ALT in group B was slightly greater (A vs $\mathrm{B},-6(-28.75,6)$ vs $-16(-52.5,-0.5)$, $P=0.291)$. The above results suggest that patients with $\mathrm{CHB}$ DH syndrome have potentially more sustained liver function damage than those with SD syndrome.

Persistent liver damage is more likely to be accompanied with HBV-related cirrhosis and hepatocellular carcinoma. In this study, the AFP level of patients with DH syndrome had a higher trend than that of the SD syndrome (Table 1, $P=0.065)$. In the dynamic observation, the improvement trend of FIB- 4 and APRI scores of patients with sustained SD syndrome showed also a better trend than patients with TCM syndrome converted from SD to DH (Table 2, $P=0.09$ and $P=0.052$, respectively), and there was no difference in the usage of antifibrosis drugs between the two groups. Studies also pointed out that CHB patients with DH syndrome were more likely to be complicated with hepatitis B cirrhosis and liver cancer. The hepatitis B cirrhosis patients with $\mathrm{DH}$ syndrome are distributed in Child-Pugh grades A, $\mathrm{B}$, and $\mathrm{C}$, but are mainly in the $\mathrm{B}$ and $\mathrm{C}$ grades and more prone to having complications, such as upper gastrointestinal hemorrhage and hepatic encephalopathy [23]. The DH syndrome (accounting for 30.97\%) also was the most common type in the III stage of liver cancer [24]. Combined with our results, it is suggested that patients with $\mathrm{CHB} \mathrm{DH}$ syndrome are associated with higher liver damage and potentially higher risk of liver fibrosis and hepatocellular carcinoma.

Medication also has a great effect on liver function, so we compared the difference in medication between the two groups of patients. In the first part of the cross-sectional study, $\mathrm{CHB}$ patients with $\mathrm{DH}$ syndrome had significantly higher level of ALT, AST, and TBA, but took more immunomodulators and hepatoprotective drugs than the SD syndrome group $(P=0.021$ and $P=0.081)$. In the second part of the dynamic follow-up observation, there was no difference between the medications of the patients in group $\mathrm{C}$ and group $\mathrm{D}$, but the rate of taking traditional Chinese herbal medicine in group A was significantly lower than that in group B, and ALT had a poor improvement trend in group A (Additional file 1). Therefore, in order to further clarify the influence of syndromes and medication information on the improvement of ALT, we conducted the logistic analysis to screen for risk factors that affect the improvement of ALT. The regression analysis results showed that the decrease of ALT is related to the syndrome types; $\mathrm{DH}$ syndrome is an adverse effect on the reduction of ALT in $\mathrm{CHB}$ patients. However, due to the small difference in medication between the two groups, the drugs were not statistically significant in models.

The biological basis of CHB patients with $\mathrm{DH}$ syndrome having a higher level of ALT is still unclear; some researches suggest that the expression of inflammatory cytokines of $\mathrm{CHB}$ patients with $\mathrm{DH}$ syndrome is relatively higher and the immune response is hyperactive. The $\mathrm{CHB}$ patients with $\mathrm{DH}$ 
syndrome had a significantly higher level of serum MIP-1 $\alpha$ than that of patients with liver-kidney yin deficiency syndrome [25]. In addition, the A allele proportion of TNF$\alpha$-308 gene in patients with $\mathrm{DH}$ syndrome of hepatitis $\mathrm{B}$ cirrhosis is significantly higher than that of non-DH syndrome [26]. Yinchenhao Decoction (YCHD) is a classic prescription for treating jaundice with $\mathrm{DH}$ syndrome. Network pharmacological studies have shown that YCHD can treat chronic liver diseases through functional modules such as immune response, inflammation, energy metabolism, and so on. However, compared with Huangqi decoction for qi deficiency syndrome, YCHD has unique functional modules related leptin-induced complement pathway and leukocyte migration across the endothelium, which may correlate with the anti-inflammatory effects [27].

\section{Limitations}

There are still some problems in this study. For example, this is a cross section study with a low level of evidence. The number of research cases is not enough, especially in the dynamic analysis. Therefore, we need to constantly expand the sample size to verify and modify the results.

\section{Conclusion}

In this study, we explored the clinical and medication differences of $\mathrm{CHB}$ patients with two common TCM. Based on the analysis of combined medication in the real world, we found that $\mathrm{CHB}$ patients with $\mathrm{DH}$ syndrome have potentially more serious and sustained liver function damage than those with SD syndrome. The DH syndrome is a negative factor for reducing serum ALT level in CHB patients. This study provides a reference for the personalized management and treatment of chronic hepatitis $B$ patients from the perspective of TCM syndromes.

\section{Data Availability}

The data used to support the findings of this study are available from the corresponding author upon request.

\section{Conflicts of Interest}

The authors declare that they have no conflicts of interest.

\section{Authors' Contributions}

Qiao-Hong Liu and Bin-Bin Zhang contributed equally to this work. Y Zhao and YY Hu conceived and designed the study. Y Zhao, L Xu, XP Shen, and YM Hai performed data collection. QH Liu and BB Zhang performed data analysis. $\mathrm{QH}$ Liu wrote the draft manuscript. Y Zhao revised the manuscript.

\section{Acknowledgments}

This study was supported by grants from the National Major Science and Technology Projects of China (grant number: 2017ZX09304002), the National Natural Science Foundation of China (grant number: 81830119), and the Science and Technology Innovation Action Plan of Shanghai Science and Technology Commission (grant number: 19401970300). The authors thank the subjects who participated in this study for providing relevant information.

\section{Supplementary Materials}

Additional file 1 (Supplementary Table 1. Comparison of clinical index difference and medication between group A and group B). Additional file 2 (Diagnostic criteria for damp-heat and spleen deficiency syndrome differentiation of CHB patients). (Supplementary Materials)

\section{References}

[1] Polaris Observatory Collaborators, "Global prevalence, treatment, and prevention of hepatitis $B$ virus infection in 2016: a modelling study," The Lancet. Gastroenterology \& Hepatology, vol. 3, no. 6, pp. 383-403, 2018.

[2] H. Wang, P. Men, Y. Xiao et al., "Hepatitis B infection in the general population of China: a systematic review and meta-analysis," BMC Infectious Diseases, vol. 19, no. 1, p. 811, 2019.

[3] Y. Xie, "Hepatitis B virus-associated hepatocellular carcinoma," Advances in Experimental Medicine and Biology, vol. 1018, pp. 11-21, 2017.

[4] World Health Organization, Guidelines for the Prevention, Care and Treatment of Persons with Chronic Hepatitis B Infection, World Health Organization, Geneva, Switzerland, 2015.

[5] F. H. Qi, Z. X. Wang, P. P. Cai, L. Zhao, J. J. Gao, and N. Kokudo, "Traditional Chinese medicine and related active compounds: a review of their role on hepatitis B virus infection," Drug Discoveries and Therapeutics, vol. 7, no. 6, pp. 212-224, 2013.

[6] T.-Y. Tsai, T.-H. Hung, H. Livneh, I.-H. Lin, M.-C. Lu, and C.-C. Yeh, "Chinese herbal medicine therapy and the risk of mortality for chronic hepatitis B patients with concurrent liver cirrhosis: a nationwide population-based cohort study," Oncotarget, vol. 9, no. 26, pp. 18214-18223, 2018.

[7] T.-Y. Tsai, H. Livneh, T.-H. Hung, I.-H. Lin, M.-C. Lu, and C.-C. Yeh, "Associations between prescribed Chinese herbal medicine and risk of hepatocellular carcinoma in patients with chronic hepatitis B: a nationwide population-based cohort study," BMJ Open, vol. 7, no. 1, Article ID e014571, 2017.

[8] L. Zhang, G. Wang, W. Hou, P. Li, A. Dulin, and H. L. Bonkovsky, "Contemporary clinical research of traditional Chinese medicines for chronic hepatitis B in China: an analytical review," Hepatology, vol. 51, no. 2, pp. 690-698, 2010.

[9] X.-X. Zeng, Z.-X. Bian, T.-X. Wu, S.-F. Fu, E. Ziea, and W. T. C. Woon, "Traditional Chinese medicine syndrome distribution in chronic hepatitis B populations: a systematic review," The American Journal of Chinese Medicine, vol. 39, no. 6, pp. 1061-1074, 2011.

[10] G. Mao, G. Cai, B. Chen, and L. Liao, "Distribution characteristics of TCM syndromes of chronic hepatitis B based on the clinical literature in the past five years," Henan Tradit Chin Med (Henan Zhong Yi), vol. 36, no. 11, pp. 1931-1933, 2016, in Chinese. 
[11] E.-L. Liu and X.-J. Wang, "Correlation between distribution of TCM syndromes and clinical test indicators based on 451 cases chronic viral hepatitis type B," China Journal of Traditional Chinese Medicine and Pharmacy (Zhong Hua Zhong Yi Yao Za Zhi), vol. 34, no. 4, pp. 1793-1795, 2019, in Chinese.

[12] L. Zhang, H. Jiang, and H. Pan, "Relativity research on chronic hepatitis B TCM syndromes and clinical test indexes," Journal of Zhejiang Chinese Medical University (Zhejiang Zhong Yi Yao Da Xue Xue Bao), vol. 36, no. 1, pp. 21-22, 2012, (in chinese).

[13] B. Chen, G. Mao, and G.-X. Cai, "Correlation between hepatobiliary damp-heat syndrome of chronic hepatitis B and ALT \& TBil: a meta-analysis," Chinese Journal of Integrated and Traditional West Medicine Liver Disorders (Zhongguo Zhong Xi Yi Jie He Gan Bing Za Zhi), vol. 24, no. 3, pp. 177-181, 2014, in Chinese.

[14] Q. Ma, "Correlation analysis between TCM Syndromes of chronic hepatitis B and clinical examination index," Clinical Journal of Chinese Medicine (Zhong Yi Lin Chuang Yan Jiu), vol. 8, no. 3, pp. 22-23, 2016, in Chinese.

[15] A. S. F. Lok and B. J. McMahon, "Chronic hepatitis B," Hepatology, vol. 45, no. 2, pp. 507-539, 2007.

[16] Chinese Society of Hepatology Chinese Medical Association, Chinese Society of Infectious Diseases, and Chinese Medical Association, "Guideline on prevention and treatment of chronic hepatitis B in China (2005)," Chinese Medical Journal (England), vol. 120, no. 24, pp. 2159-2173, 2007.

[17] Association Internal Medicine Hepatopathy Committee of Chinese Traditional Medicine, "The standards of TCM differential syndromes of viral hepatitis," Traditional Chinese Medicine, vol. 5, pp. 39-40, 1992, in Chinese.

[18] X. Y. Zheng, Guideline for Clinical Research on New Chinese Medicine Drugs, Chinese Medical Sciences Technology, Beijing, China, 2002.

[19] G. Mao, G. Cai, and B. Chen, "The TCM syndrome distribution characteristic of 1868 chronic hepatitis B patients and the correlation analysis with virology indices of serum by logistic regression," Journal of Traditional Hunan University of Chinese Medicine (Hunan Zhong Yi Yao Da Xue Xue Bao), vol. 34, no. 4, pp. 24-28, 2014, in Chinese.

[20] Y. Liu, "The study of relationship of traditional Chinese medicine type of syndrome of Autoirronune hepatitis and objective detective signs," Master Dissertation, Guangzhou University of Chinese Medicine (Guangzhou Zhong Yi Yao Da Xue), 2016, in Chinese.

[21] L.-M. Gu, P.-R. Cao, C. Gu, L.-F. Wei, and Y.-Z. Tian, "Correlation between traditional Chinese medicine syndrome and clinical biochemical indexes of non-alcoholic fatty liver," Journal of Nanjing University Traditional Chinese Medicine (Nanjing Zhong Yi Yao Da Xue Xue Bao), vol. 35, no. 6, pp. 738-740, 2019, in Chinese.

[22] M. G. Ghany, J. J. Feld, K.-M. Chang et al., "Serum alanine aminotransferase flares in chronic hepatitis B infection: the good and the bad," The Lancet Gastroenterology \& Hepatology, vol. 5, no. 4, pp. 406-417, 2020.

[23] C. Hu, "Correlation analysis of TCM syndrome types of hepatitis B cirrhosis and child-pugh classification," Guangming Journal of Chinese Medicine (Guangming Zhong Yi), vol. 33, no. 4, pp. 465-466, 2018, in Chinese.

[24] G. Yang, "Study on diagnosis and treatment rule of primary liver cancer based on data mining," Doctoral Dissertation, Hubei University of Chinese Medicine (Hubei Zhong Yi Yao Da Xue), 2019, in Chinese.
[25] Y.-Y. Lu, Y. Zhao, Y.-N. Song et al., "Serum cytokine profiling analysis for zheng differentiation in chronic hepatitis B," Chinese Medicine, vol. 10, no. 1, p. 24, 2015.

[26] Z.-P. Shi, T.-Y. Wu, Y. Liu et al., "Research on the association between hepatitis B liver cirrhosis of dampness-heat syndrome and tumor necrosis factor- $\alpha$ gene polymorphism," China Journal of Traditional Chinese Medicine and Pharmacy (Zhong Hua Zhong Yi Yao Za Zhi), vol. 29, no. 6, pp. 20042006, 2014, in Chinese.

[27] Z. Chen, X. Wang, Y. Li et al., "Comparative network pharmacology analysis of classical TCM prescriptions for chronic liver disease," Frontiers in Pharmacology, vol. 10, p. 1353, 2019. 